\title{
What is the most appropriate treatment for chronic rhinosinusitis?
}

\author{
Abigail Walker, ${ }_{1}^{\oplus 1}$ Carl Philpott, $^{\oplus 2}$ Claire Hopkins $^{1}$
}

'Guy's and Saint Thomas' Hospitals NHS Trust, London, UK

${ }^{2}$ James Paget University Hospitals NHS Foundation Trust, Great Yarmouth, UK

\section{Correspondence to}

Abigail Walker, Guy's and Saint Thomas' Hospitals NHS Trust, London, UK;

abigail.walker@nhs.net

Received 5 March 2019

Accepted 22 June 2019

Published Online First 10 July 2019

Check for updates

(c) Author(s) (or their employer(s)) 2019. No commercial re-use. See rights and permissions. Published by BMJ.

To cite: Walker A, Philpott C, Hopkins C. Postgrad Med J 2019:95:493-496.

\section{ABSTRACT}

Chronic rhinosinusitis (CRS) is a common, treatable disease that affects approximately $11 \%$ of British adults. It places an enormous burden on patients, with significant detriment to their quality of life, and the health service as it consumes vast numbers of both primary and secondary care resources. However, there is considerable variability in treatment strategies and prescribing practices. This review summarises the key recommendations from landmark guidelines in the treatment of CRS and critically appraises the evidence for treatment.

Chronic rhinosinusitis (CRS) is defined as inflammation of the mucosal lining of the nasal cavity and paranasal sinuses that has been present for more than 3 months, causing characteristic symptoms such as nasal blockage, discharge, reduction or loss of smell sensation and facial pressure. It is a broad clinical description that encompasses several phenotypes, most often divided into CRS with polyps and CRS without polyps. CRS may be the endpoint of several different inflammatory pathways and may be secondary to specific conditions such as cystic fibrosis or fungal aetiology, but most often cannot be attributed to a single pathophysiological trigger.

CRS places an enormous burden on both physicians and its sufferers. It is common among the general population, affecting approximately $11 \%$ of UK adults, ${ }^{1}$ and causes greater detriment to quality of life $(\mathrm{QoL})$ than angina or chronic lower respiratory tract disease. ${ }^{2}$ Not only is it a QoL issue, patients with CRS frequently have comorbid asthma, and poorly controlled CRS is associated with more frequent asthma exacerbations and a need for hospitalisation. The socio-economic cost of CRS is significant with $57 \%$ of patients reporting absenteeism and 28\% experiencing associated anxiety and depression. ${ }^{3}{ }^{4}$ One per cent of all UK adults will visit their general practitioner (GP) with CRS symptoms each year and require an average of four appointments each year; despite this, one in three patients in primary care has poorly controlled symptoms. ${ }^{5}$ CRS that cannot be adequately managed in primary care may require referral to secondary care, and data from Hospital Episode Statistics show that in a single year there were approximately 120000 outpatient consultations for CRS and 40000 sinus operations performed in England and Wales. ${ }^{6}$ However, there remains considerable debate regarding optimum management strategies leading to wide variation in practice. ${ }^{7}$

\section{TREATMENT EVIDENCE AND GUIDELINES}

The touchstone for treatment of CRS is The European Position Paper on Rhinosinusitis and Nasal Polyps published in 2012 (EPOS 2012). ${ }^{8}$ EPOS 2012 syntheses the best available evidence and expert opinion into easy to follow treatment flowcharts for both primary care physicians and ear, nose and throat specialists, but acknowledges the large gaps in highquality trial data on which to base their guidance. In the intervening 7 years since EPOS was published, the International Consensus Statement on Allergy and Rhinology (ICAR) ${ }^{9}$ was agreed by a multinational working group who undertook a comprehensive overview of the literature. The working group graded the available evidence and used it as the basis for recommendations; where no evidence was available, no recommendation was made. ICAR is therefore an exhaustive insight into the current state of the science in CRS and an invaluable tool for the otolaryngology community; for primary care, however, the EPOS 2012 remains perhaps the more accessible guide. In addition, there have also been several Cochrane reviews ${ }^{10-16}$ of treatments available for CRS, and these reviews along with the international consensus guidelines have been used to synthesise a National Institute for Health and Care Excellence (NICE) clinical knowledge summary, most recently updated in $2018 .{ }^{17}$

\section{Intranasal treatment}

The foundation of CRS treatment is regular use of intranasal saline irrigation and corticosteroids and this strategy finds unanimous favour among the guidelines mentioned above. The evidence is weak but consistent: a Cochrane systematic review studied the effects of saline irrigation in people with chronic sinusitis. ${ }^{12}$ This identified only two studies that could robustly be included - one which compared the effect of high volume hypertonic saline irrigation to placebo in patients with CRS both with and without polyps, and another which studied only patients with polyps and compared the use of nasal saline irrigation. This Cochrane review concluded that there was weak evidence from these trials to support the use of nasal saline irrigation. In addition, it is a low-cost treatment which with low risk of adverse events. For this reason, it is recommended for virtually all patients with CRS although in practice is usually used in combination with other therapies.

Intranasal corticosteroids are similarly favoured by all expert guidelines. There is a greater number of trials on which to base this opinion. A Cochrane systematic review ${ }^{11}$ identified (search date August 2015) 18 randomised controlled trials which studied the use of intranasal steroids versus placebo 
in CRS. The majority of these studies included patients with nasal polyps. Regular intranasal corticosteroid use was reported to be superior to placebo in all reported symptoms, although there was no evidence to support an overall improvement in QoL. A further Cochrane review of the different formulations of intranasal corticosteroids and methods of delivery ${ }^{13}$ did not identify any single agent with convincing evidence of superiority; however, there is an advantage offered by the second-generation agents (eg, mometasone furoate, fluticasone propionate, fluticasone furoate) which have favourable pharmacokinetic characteristics that minimise systemic bioavailability $(<1 \%)$ compared with older intranasal corticosteroids (eg, beclomethasone diproprionate, betamethasone).

\section{Oral corticosteroids}

Oral corticosteroids are commonly prescribed in the treatment of CRS although there is a distinct lack of evidence for their efficacy. The NICE Clinical Knowledge Summary (CKS) makes no reference to their use. A Cochrane review in $2016^{16}$ identified no studies of their use in CRS without polyps, a fact which is also reflected in the ICAR guideline. ${ }^{9}$ Their use in patients with polyps is better established, with several studies demonstrating short-term efficacy of oral corticosteroids in improving CRS-related QoL scores. Treatment courses are short-a typical regime might be $30 \mathrm{mg}$ per day of prednisolone for 2-3 weeks - and thus the risk of side effects such as adrenal suppression and loss of bone mass is minimal. However, there is a risk of side effects such as gastrointestinal disturbance and insomnia even with these short courses. Patients should also be warned of the risk of osteonecrosis-a risk which may occur at doses of steroid previously thought to be safe (lowest reported associated total dose $105 \mathrm{mg}$, median associated dose $981 \mathrm{mg}$ ). ${ }^{18}$ The benefit of oral corticosteroids typically last 3-6 months, and so are a reasonable choice for patients who have not improved on the first line of saline irrigation and topical corticosteroids. However, an evidence-based risk analysis of oral corticosteroid use in CRS with polyps performed by Leung et $a l^{19}$ demonstrated that risk exceeded benefit if used more frequently than once every 2 years. Oral corticosteroid use is therefore restricted to short, infrequent courses, and with full disclosure of the potential serious associated side effects for patients.

\section{Antibiotics}

Where there is consensus recommending the use of nasal douches and corticosteroids, there is controversy regarding the use of antibiotics. Antibiotics are one of the most commonly prescribed medicines for CRS: of the 1\% of UK adults with CRS who visit their GP each year, 91\% of them will receive an antibiotic prescription. ${ }^{20}$ They will often receive not just one but repeated courses of antibiotics. ${ }^{21}$ There is pressure on GPs to use antibiotics as a first-line treatment, as recently some Clinical Commissioning Groups have insisted on a 3-month trial of macrolide antibiotics prior to secondary care referral. ${ }^{22}$

With the exception of acute infective exacerbations, routine use of antibiotics is controversial for three main reasons: first; the lack of high-quality evidence to support their efficacy; second, the emerging threat of antibiotic resistance and third, the side effects which may accompany some of the most commonly used agents.

In 2016, the ICAR guideline noted a paucity of evidence for antibiotic use ${ }^{9}$; however, on balance it recommended that patients with polyps should not be prescribed short courses of non-macrolide antibiotics, unless in the particular circumstances of suffering an acute exacerbation. No conclusions could be drawn on longer courses, or for patients without polyps. This echoes the findings of a Cochrane review of the use of antibiotics in CRS, which found very little evidence that non-macrolide antibiotics are effective in patients with CRS although only two studies were fit for inclusion in analysis. ${ }^{15}$ These recommendations guided the NICE $\mathrm{CKS}^{17}$ to make the recommendation to seek specialist advice before initiating long-term antibiotics in primary care.

It is, however, recognised that there does remain a role for appropriate use of macrolide antibiotics in specific circumstances. In particular, there is growing interest in immune-modulating and anti-inflammatory effects of macrolides in chronic airway inflammatory disease, with low-dose long-term macrolides being prescribed for its effects on the immune response and not primarily as an anti-bacterial agent. ${ }^{23}$ The Cochrane review of 2016 noted that there was a moderate quality of evidence of a modest improvement in disease-specific QoL in patients with CRS without nasal polyps receiving 3 months of a macrolide. ${ }^{15}$ However, this improvement was short-lived, as 3 months following the cessation of the antibiotics there was no measurable effect of treatment.

\section{Resistance}

Antibiotic resistance is considered one of the most significant threats to patients' safety in Europe. ${ }^{24}$ Evaluating effectiveness of antibiotics and promoting appropriate usage is integral to the UK 5-year antimicrobial resistance strategy. ${ }^{25}$ Given the high prevalence of CRS and the variability in prescribing, overuse of inappropriate antibiotics may represent a public health danger through selective pressure on bacteria and resistance. In particular, macrolide resistance has been noted to have implications in infections secondary to Streptococcus pneumoniae (upper and lower respiratory tract infections) and Mycoplasma genitalium (sexually transmitted infections), leading to longer duration of illness and requirement for multiple antibiotic courses. ${ }^{26}$

\section{Side effects}

Antibiotics are commonly associate with wide range of side effects ranging from the mild (rash, nausea, diarrhoea) to the life threatening (anaphylaxis). Specific to the use of macrolides are concerns about potential cardiovascular side effects. ${ }^{27}$ There have been a number of publications raising concerns about cardiac toxicity with erythromycin in patients with a prolonged QT interval. ${ }^{28}{ }^{29} \mathrm{~A}$ 2016 study which looked at adverse outcomes associated with clarithromycin use found a significant association was found between clarithromycin use and cardiovascular mortality, where longer durations of clarithromycin use were associated with more cardiovascular events. ${ }^{29}$ By contrast, this association was not found with use of $\beta$-lactam antibiotics leading the authors to hypothesise that it was an effect specific to clarithromycin. This hypothesis was unintentionally tested by the lead investigators of the CLARICOR trial (Effect of Clarithromycin on Mortality and Morbidity in Patients With Ischemic Heart Disease) who had planned to measure the efficacy of a 2-week course of $500 \mathrm{mg}$ clarithromycin two times per day to improve the clinical manifestations of stable coronary heart disease. ${ }^{30}$ The study was halted prematurely due to the finding of excess deaths in the intervention group: all-cause mortality was significantly higher in the group had taken clarithromycin, an effect which persisted for 10 years post-intervention. The use of clarithromycin in patients with known cardiovascular disease is therefore only undertaken with a significant degree of caution.

\section{Surgery}

Patients who are referred to secondary care who are considered to have tried and failed appropriate medical therapy - which is to say, a sufficient trial of nasal douching and topical steroids-are considered candidates for endoscopic sinus surgery (ESS). ${ }^{31} 32$ ESS is a loosely defined procedure and can range from very 
simple balloon expansion of the sinus openings, to extensive procedures that open every bony partition within the sinuses. The risks of surgery include epistaxis, breach of the skull base with resultant CSF leak or meningitis and injury to the intraorbital structures which may result in impaired vision. Insufficient evidence to define the role and extent of surgery contributes to a fivefold variation in UK intervention rates, ${ }^{33}$ with wide variation in surgical practice and very high rates of revision surgery. ${ }^{34} 35$ Such uncertainty resulted in inclusion of ESS in the NICE Database of Treatment Uncertainties. ${ }^{36}$

However, there can be no doubt that in appropriately selected patients, surgery offers significant and durable relief from symptoms. The largest UK study of CRS and its outcomes demonstrated that not only was there a significant improvement in patient-reported symptoms following ESS, but that patients who had surgery earlier in their disease course had a greater degree of improvement that lasted longer versus those who underwent delayed surgery. ${ }^{37}$ In addition to improving nasal symptoms, a systematic review demonstrated that ESS is associated with improved asthma control with significantly fewer attacks, hospitalisations, and a reduced requirement for medication use following sinus surgery in patients with comorbid CRS and asthma. ${ }^{38}$ Each of the international consensus guidelines referenced above recommends that patients who have tried a course of appropriate medical therapy but do not have control of their symptoms should be considered as a candidate for surgery. ${ }^{89}$

\section{THE PATH FORWARDS}

In the last two decades, the majority of new treatments that have become available for CRS have in the main been refinements rather than revolutions-for example, new formulations of intranasal steroids or third-generation antihistamines. However, there are some genuinely new and exciting innovations which are on the cusp of integration to clinical practice. Monoclonal antibodies that bind to targets such as IgE, interleukin-4 (IL-4) and IL-5 were developed primarily as treatments for asthma, but clinical trials have demonstrated that several of these agents may also be effective in CRS with polyps. ${ }^{39}$ At present, they remain expensive and are limited to subsets of patients with difficult to treat asthma, but as prices fall in future and evidence for their efficacy accumulates then in future they may become available for the treatment of CRS. There have also been several promising avenues of research that may unlock further therapeutic targets, such as the role of the microbiome in sinus disease. However, as alluded to in the many international guidelines, there is a paucity of evidence for many of the treatments that are currently used in CRS before even considering new innovations. This has led to a redoubling of efforts in CRS research to produce high-quality evidence for commonly available treatments. Key to these efforts is the need to accurately define the endotypes of disease which will be studied in trials, as broad clinical phenotypes such as 'polyps' may encompass a wide range of underlying pathophysiology. Tightly defining the endotypes of disease for trial inclusion will permit robust examination of the response to treatment in each subgroup. Meta-analyses of CRS outcomes have been hampered by the broad clinical phenotypes, and using precise endotypes will enhance our ability to pool data from multiple trials; this will also be enabled by international consensus statements that define key criteria such appropriateness for ESS ${ }^{40}$ and standardised sets of outcome measures; ${ }^{41}$ and funding has been secured for one of the largest ever trials of interventions in CRS which will specifically examine the question of the efficacy of both macrolides and ESS (the MACRO trial ${ }^{42}$ ) in subgroups of patients defined by precise clinical and pathological parameters.
Main messages

Chronic rhinosinusitis (CRS) is a common disease in UK adults and causes significant detriment to life.

- The best evidence for effective treatment is for topical intranasal corticosteroids and saline irrigation.

- For patients who do not respond to this first-line treatment, there are many potential avenues for further treatment but there is controversy about which strategy is the most effective

\section{Current research questions}

- Is it possible to identify specific endotypes of CRS to replace the broad clinical phenotypes that we currently base our treatment plans on?

- Are macrolide antibiotics an effective treatment in CRS?

- Is best medical treatment superior to surgery in the treatment of CRS?

\section{Key references}

- Fokkens WJ, et al, European Position Paper on Rhinosinusitis and Nasal Polyps 2012. Rhinolgy 20123 p preceding table of contents, 1-298.

- Orlandi RR, et al, International Consensus Statement on Allergy and Rhinology: Rhinosinusitis. Int Forum Allergy Rhinol 2016. 6 Suppl 1: p. S22-209.

- NICE. Sinusitis. 2018; Available from: https://cks.nice.org.uk/ sinusitis\#!scenario:1

- Head K, et al, Systemic and topical antibiotics for chronic rhinosinusitis. Cochrane Database Syst Rev 2016. 4: p. CD011994

- Hopkins C, et al, Long-term outcomes from the English national comparative audit of surgery for nasal polyposis and chronic rhinosinusitis. Laryngoscope 2009. 119 : p. 2459-65.

Self-assessment questions

1. Which of the following are typical symptoms of chronic rhinosinusitis? (a) Bleeding and crusting (b) Unilateral blood tinged discharge (c) Blockage, discharge, loss of sense of smell, facial pressure (d) Visual disturbance

2. The first-line treatment for chronic rhinosinusitis (CRS) is oral steroids and antibiotics. True or false?

3. A prolonged course of antibiotics (eg, up to 3 months) may occasionally be used in specific circumstances. What group of antibiotics has the best evidence to support their use for prolonged courses in CRS? (a) Penicillins (b) Macrolides (c) Aminoglycosides (d) Fluoroquinolones

4. And what adverse effects should the patient be advised of with these long-term antibiotics? (a) Cardiac comorbidity (b) Osteonecrosis of the femoral head (c) Epistaxis (d) Infertility

5. There is good evidence to support the superiority of surgical treatment of CRS compared with medical treatment. True or false? 
At present, treatment of CRS exists in a time of genuine clinical equipoise where treatment is empirical and even international guidelines depend more heavily on expert opinion than robust scientific statements. Ongoing research endeavours aim to transform the treatment of CRS and usher in the era of precise, personalised medicine for these patients.

Contributors All three authors contributed equally to this article with review of evidence, writing the text, and editing the whole piece.

Funding The authors have not declared a specific grant for this research from any funding agency in the public, commercial or not-for-profit sectors.

Competing interests $\mathrm{CH}$ and $\mathrm{CP}$ are the chief investigators of the NIHR funded "MACRO" trial.

Patient consent for publication Not required.

Provenance and peer review Not commissioned; externally peer reviewed.

\section{REFERENCES}

1 Hastan D, Fokkens WJ, Bachert C, et al. Chronic rhinosinusitis in Europe--an underestimated disease. A GA22LEN study. Allergy 2011;66:1216-23.

2 Gliklich RE, Metson R. The health impact of chronic sinusitis in patients seeking otolaryngologic care. Otolaryngol Head Neck Surg 1995;113:104-9.

3 Sahlstrand-Johnson P, Ohlsson B, Von Buchwald C, et al. A multi-centre study on quality of life and absenteeism in patients with CRS referred for endoscopic surgery. Rhinology 2011:49:420-8.

4 Erskine SE, Hopkins C, Clark A, et al. Chronic rhinosinusitis and mood disturbance. Rhin 2017:55:113-9.

5 Lange B, Holst R, Thilsing T, et al. Quality of life and associated factors in persons with chronic rhinosinusitis in the general population: a prospective questionnaire and clinical cross-sectional study. Clin Otolaryngol 2013;38:474-80.

6 Hospital Episode Statistics Department of Health; 2013.

7 Vennik J, Eyles C, Thomas M, et al. Management strategies for chronic rhinosinusitis: a qualitative study of GP and ENT specialist views of current practice in the UK. BMJ Open 2018;8:e22643.

8 Fokkens WJ, Lund VJ, Mullol J, et al. EPOS 2012: European position paper on rhinosinusitis and nasal polyps 2012. A summary for otorhinolaryngologists. Rhinology 2012;50:1-12.

9 Orlandi RR, Kingdom TT, Hwang PH, et al. International Consensus Statement on Allergy and Rhinology: Rhinosinusitis. Int Forum Allergy Rhinol 2016;6 Suppl 1:S22-\$209.

10 Head K, Sharp S, Chong L-Y, et al. Topical and systemic antifungal therapy for chronic rhinosinusitis. Cochrane Database Syst Rev 2018;16.

11 Chong LY, Head K, Hopkins C, et al. Intranasal steroids versus placebo or no intervention for chronic rhinosinusitis. Cochrane Database Syst Rev 2016;115.

12 Chong LY, Head K, Hopkins C, et al. Saline irrigation for chronic rhinosinusitis. Cochrane Database Syst Rev 2016;67.

13 Chong LY, Head K, Hopkins C, et al. Different types of intranasal steroids for chronic rhinosinusitis. Cochrane Database Syst Rev 2016;25(Suppl 2).

14 Head K, Chong LY, Hopkins C, et al. Short-course oral steroids as an adjunct therapy for chronic rhinosinusitis. Cochrane Database Syst Rev 2016;27.

15 Head K, Chong LY, Piromchai P, et al. Systemic and topical antibiotics for chronic rhinosinusitis. Cochrane Database Syst Rev 2016;19.

16 Head K, Chong LY, Hopkins C, et al. Short-course oral steroids alone for chronic rhinosinusitis. Cochrane Database Syst Rev 2016;2.

17 NICE. Sinusitis, 2018. Available: https://cks.nice.org.uk/sinusitis\#!scenario:1

18 Kennedy P, Bassiouni A, Psaltis A, et al. Avascular necrosis after oral corticosteroids in otolaryngology: Case report and review of the literature. Allergy Rhinol 2016;7:50-4.

19 Leung RM, Dinnie K, Smith TL. When do the risks of repeated courses of corticosteroids exceed the risks of surgery? Int Forum Allergy Rhinol 2014;4:871-6.

20 Gulliford MC, Dregan A, Moore MV, et al. Continued high rates of antibiotic prescribing to adults with respiratory tract infection: survey of $568 \mathrm{UK}$ general practices. BMJ Open 2014;4:e006245.

21 Akkerman AE, Kuyvenhoven MM, van der Wouden JC, et al. Prescribing antibiotics for respiratory tract infections by GPs: management and prescriber characteristics. $\mathrm{Br} J$ Gen Pract 2005:55:114-8.

22 Soni-Jaiswal A, Philpott C, Hopkins C. The impact of commissioning for rhinosinusitis in England. Clin Otolaryngol 2015;40:639-45.

23 Cervin A, Wallwork B. Macrolide therapy of chronic rhinosinusitis. Rhinology 2007:45:259-67.

24 Lodato EM, Kaplan W. Priority Medicines for Europe and the World "A Public Health Approach to Innovation": Background Paper 6.1 Antimicrobial resistance World Health Organisation; 2013.
25 Davies SC, Gibbens N. UK Five Year Antimicrobial Resistance Strategy 2013 to 2018 Department of Health and Department for Environment, Food \& Rural Affairs; 2013.

26 Gaynor M, Mankin AS. Macrolide antibiotics: binding site, mechanism of action, resistance. Curr Top Med Chem 2003:3:949-60.

27 Gibbins NE, Theokli C, Hopkins C. Time to reconsider guidelines on clarithromycin in chronic rhinosinusitis? BMJ 2013;346:f2678

28 Wong AYS, Chan EW, Anand S, et al. Managing Cardiovascular Risk of Macrolides: Systematic Review and Meta-Analysis. Drug Saf 2017;40:663-77.

29 Cheng Y-J, Nie X-Y, Chen X-M, et al. The Role of Macrolide Antibiotics in Increasing Cardiovascular Risk. J Am Coll Cardiol 2015;66:2173-84.

30 Winkel P, Hilden J, Hansen JF, et al. Clarithromycin for stable coronary heart disease increases all-cause and cardiovascular mortality and cerebrovascular morbidity over 10years in the CLARICOR randomised, blinded clinical trial. Int I Cardiol 2015;182:459-65.

31 Baguley C, Brownlow A, Yeung K, et al. The fate of chronic rhinosinusitis sufferers after maximal medical therapy. Int Forum Allergy Rhinol 2014;4:525-32.

32 Young LC, Stow NW, Zhou L, et al. Efficacy of medical therapy in treatment of chronic rhinosinusitis. Allergy Rhinol 2012;3:8-12.

33 Hopkins C. Commissioing Guide: Chronic Rhinosinusitis. Royal College of Surgeons of England London; 2016.

34 Philpott C, Hopkins C, Erskine S, et al. The burden of revision sinonasal surgery in the UK-data from the Chronic Rhinosinusitis Epidemiology Study (CRES): a cross-sectional study. BMJ Open 2015;5:e06680.

35 Hopkins C, Slack R, Lund V, et al. Long-term outcomes from the English national comparative audit of surgery for nasal polyposis and chronic rhinosinusitis. Laryngoscope 2009;119:2459-65.

36 NICE. Database of Uncertainties of Treatments.

37 Hopkins C, Rimmer J, Lund VJ. Does time to endoscopic sinus surgery impact outcomes in Chronic Rhinosinusitis? Prospective findings from the National Comparative Audit of Surgery for Nasal Polyposis and Chronic Rhinosinusitis. Rhinology 2015;53:10-17.

38 Vashishta R, Soler ZM, Nguyen SA, et al. A systematic review and meta-analysis of asthma outcomes following endoscopic sinus surgery for chronic rhinosinusitis. Int Forum Allergy Rhinol 2013;3:788-94.

39 Pauwels B, Jonstam K, Bachert C. Emerging biologics for the treatment of chronic rhinosinusitis. Expert Rev Clin Immunol 2015;11:349-61.

40 Rudmik L, Soler ZM, Hopkins C, et al. Defining appropriateness criteria for endoscopic sinus surgery during management of uncomplicated adult chronic rhinosinusitis: a RAND/UCLA appropriateness study. Int Forum Allergy Rhinol 2016;6:557-67.

41 Hopkins C, Hettige R, Soni-Jaiswal A, et al. CHronic Rhinosinusitis Outcome MEasures (CHROME), developing a core outcome set for trials of interventions in chronic rhinosinusitis. Rhinology 2018;56:22-32.

42 Philpott C, le Conte S, Beard D, et al. Clarithromycin and endoscopic sinus surgery for adults with chronic rhinosinusitis with and without nasal polyps: study protocol for the MACRO randomised controlled trial. Trials 2019;20:246.

\section{Answers}

1. Answer: (c) These are typical CRS symptoms. Bleeding and crusting should raise the possibility of vasculitis, septal perforations, or recreational cocaine use. Unilateral blood tinged discharge is a red flag sign that should prompt concerns of malignancy. Visual disturbance may be caused by rare complications of CRS, but is not at all typical.

2. Answer: False. The first-line treatment for CRS should be topical steroids and saline irrigation.

3. Answer: Macrolides.

4. Answer: Cardiac comorbidity, particularly in patients with known heart disease. Osteonecrosis of the femoral head is a complication of oral steroids while topical steroids may cause epistaxis. Macrolides are not recognised to cause infertility but their use should be restricted during pregnancy. The Food and Drug Administration of USA recommend that if a macrolide must be used in pregnancy then azithromycin is category B (ie, safe in animal studies) while clarithromycin is category $C$ (ie, shown to cause harm in animal studies).

5. Answer: False. There are very few high-quality trials that compare medical treatment to surgery, and meta-analysis is made difficult by wide variability in study protocols. 